\title{
Évaluation comparative de deux méthodes d'analyse de cycle de vie simplifiée dans un contexte de conception de produit au sein de PME.
}

\author{
C. Côté lokinlado@hotmail.com \\ Faculté de l'aménagement — Design industriel, Université de Montréal, Canada \\ et \\ R. P. Gouvinhas reidson@ct.ufrn.br \\ Centro de Tecnologia - CT Departamento de Engenharia de Produção - DEPT Brésil
}

\begin{abstract}
Résumé
Ce projet cherche à repérer et à évaluer différents outils d'aide à la conception de produits à moindre impact environnemental utilisables au sein de petites et moyennes entreprises (PME).
\end{abstract}

Le but de notre recherche consiste à analyser deux outils d'analyse de cycle de vie simplifiée (Environmentally Responsible Product Assesment. ERPA [3] et Ecoindicator'99 [19]) les plus utilisés dans la phase de conception. Notre choix tient au fait qu'ils sont bien documentés et que les types d'analyse qu'ils proposent sont très différents un de l'autre. Afin de réaliser cette étude, nous procéderons à l'évaluation du cycle de vie d'un produit à l'aide de ces deux méthodes. Le produit choisi est fabriqué par une PME brésilienne située dans la région du Nord-Est, dans l'État de Rio Grande do Norte.

Les résultats de cette analyse permettront d'identifier, dans le cas des PME brésiliennes, quels sont les avantages et les inconvénients liés à l'utilisation de ce type d'outil en regard de leur fiabilité, de leur accessibilité et de leur faisabilité. Finalement, nous proposerons certaines modifications, afin de rendre ces méthodes plus facilement utilisables par les PME.

\section{Introduction}

Ce projet s'inscrit dans la deuxième phase d'un projet de recherche réalisé dans le cadre des travaux de l'« Institute Fabric of Millennium - IFM », lequel est un projet de recherche scientifique nationale cherchant à donner un support aux compagnies et PME brésiliennes dans le domaine de la production de produit. Ce projet est également soutenu par le CNPq (Conselho Nacional de Pesquisa) $[1,2]$. Cette phase du projet a été réalisée en coopération avec le laboratoire - écodesign affilié au groupe de recherche le CIRAIG [15].

$\mathrm{Ce}$ projet de recherche a pour objectif d'évaluer l'utilisation d'outils d'analyse de cycle de vie simplifiée en éco-conception au sein de petites et moyennes entreprises brésiliennes pendant la phase de conception.

Depuis la dernière décennie, le souci environnemental et la concurrence féroce sont devenus des enjeux incontournables que les compagnies et particulièrement les PME ont du considérer afin de pouvoir rester actives dans l'actuel contexte de globalisation des marchés. La plupart des compagnies et PME brésiliennes sont considérées comme étant des entreprises familiales. $\mathrm{Au}$ Brésil, 60 millions d'individus travaillent pour plus de 4,5 millions de petites entreprises, dont $98 \%$ excelle dans le secteur industriel, commercial, et de services. De plus, il est à noter que ce type d'entreprises est à l'origine de $60 \%$ des travaux urbains et de $21 \%$ du PIB, tout en totalisant $12.4 \%$ de toutes les exportations nationales [14].

Le présent projet de recherche vise ainsi à démontrer que l'inclusion de la notion d'éco-conception et des outils d'analyse connexe dans la méthode de production des PME aurait, pour ces dernières, une incidence positive sur leur positionnement sur les marchés ainsi que sur leur croissance.

\section{Choix du terrain d'étude.}

L'entreprise choisie, Meubles Jales [20], est présente sur le marché du meuble brésilien depuis plus de 40 ans. Dès son arrivée sur le marché, elle a su offrir à sa clientèle des meubles fonctionnels et de qualité. En 1961, José Jales Sobrinho créa les Meubles Jales. L'entreprise oriente ses efforts sur la production de meubles (personnalisés ou préfabriqués) qui répondent aux demandes spécifiques de leurs clients.

Les principaux matériaux utilisés dans la fabrication des meubles Jales sont les panneaux de fibre de moyenne densité (MDF) et les bois d'essence naturelle. Les principaux procédés de fabrication utilisés par l'entreprise sont: la transformation des matériaux prêts pour la production, le pré traitement des surfaces et la peinture, l'assemblage et l'emballage. De plus, l'entreprise assure 
la livraison de sa production dans des points de vente et chez certains de ses clients. Le marché exploité par Moveis jales est un marché qui est entièrement local.

En 2003, José Jales Sobrinho, fit face à d'énormes problèmes financiers. Afin de redonner un second souffle à son entreprise, il accepta de participer au projet proposé par le groupe de recherche du département d'ingénierie de production de l'Universidade Federal do Rio Grande Norte l'UFRN, Potidesign [21]. Dans la cadre du projet Potidesign, une série d'objectifs et d'action ont été mis en place afin d'aider l'entreprise à se redresser.

\section{Méthode}

Méthode d'analyse

Deux méthodes d'analyse de cycle de vie simplifiée (ACVS), ERPA et Éco-indicateur99, ont été examinées lors d'une étude de cas menée au moment de la conception, par la PME, d'une table d'ordinateur. L'essentiel des données a été collecté lors d'une recherche empirique menée au sein de l'entreprise. Les autres données utilisées ont été tirées d'ouvrages consultés ainsi que d'informations recueillies sur les sites Web des fournisseurs. Nous croyons important de spécifier que l'analyse avec ERPA et Éco-indicater99 a été effectuée indépendamment et sans connaissance des résultats.

Une fois les objectifs, le champs d'étude, ainsi que les frontières du système définies, une attention particulière $a$ été portée aux trois autres phases de l'évaluation d'impact environnemental soit: l'analyse d'inventaire, l'évaluation des impacts et l'interprétation. C'est principalement en fonction de ces trois points que s'orientera notre analyse comparative.

Après l'exécution des analyses à l'aide des outils retenus, nous avons comparé les résultats. Notre analyse comparative a été réalisée à partir du questionnaire suivant dont certaines questions ont été tirées de l'article de Hochschorner1 E. et Finnveden, G. [16]

Le choix des questions repose sur leur capacité à illustrer la pertinence des caractéristiques des méthodes.

1. Quel est le temps nécessaire pour la réalisation de l'analyse?

2. Selon le contexte de l'étude, la méthode était-elle appropriée?

3. Est-ce que la méthode est facilement comprise par l'évaluateur?

4. Est-il possible d'inclure des informations qualitatives ou quantitatives lors de l'analyse?

5. La méthode est-elle facile a utiliser lors de cette phase de l'analyse?

6. La réalisation de cette phase de l'analyse se fait-elle rapidement?

7. La méthode permet-elle une flexibilité? L'utilisateur peut-il l'ajuster pour répondre à ses besoins?

8. La méthode permet-elle de limiter la collecte de données lors de la phase d'inventaire?

9. L'étape de collecte de donnée lors de l'étape d'inventaire permet de tenir compte des données bibliographiques ou génériques?

10. Le mode et les outils de collecte de données proposées par cette méthode sont-ils efficaces?

11. La description du système produit est-elle simple à faire?

12. Les données requises par la méthode sont-elle simples à obtenir?
13. Est-ce que la méthode utilise un mode de calcul qui permet à l'évaluateur d'arriver à une valeur unique en fin d'analyse?

14. La réalisation de cette phase de l'analyse se fait-elle rapidement?

15. La méthode permet-elle une flexibilité. L'utilisateur peut-il l'ajuster pour répondre à ses besoins?

16. La méthode permet-elle une analyse qui tient compte de plusieurs critères (matières, ressources; énergie; l'effet de serre; eutrophisation) et ce, tout au long du cycle de vie de l'objet?

17. Quel est le temps requis pour la réalisation de cette phase de l'analyse?

18. La méthode permet-elle une flexibilité. L'utilisateur peut-il l'ajuster pour répondre à ses besoins?

19. Les conclusions de l'analyse sont-elles fiables?

20. La méthode permet-elle d'arriver à un résultat convaincant? Et la méthode permet-elle d'identifier les impacts majeurs générés par le produit évalué?

\section{Méthodes ACVS}

Le choix des méthodes d'analyse s'est fait suite à une synthèse de la littérature existante sur les outils d'analyse de cycle de vie simplifiée utilisés dans la conception de produits écologiques :

Depuis ces dernières années, beaucoup d'efforts ont été investis pour mettre au point des outils d'analyse de cycle de vie $(\mathrm{ACV})$ et des ACVS. Ces efforts ont eu pour résultat d'harmoniser les méthodes d'évaluation d'impact environnemental entre leurs divers utilisateurs: les designers industriels, les manufacturiers, les responsables des programmes d'étiquetage environnemental, les gouvernements, etc.

Sachant qu'une Analyse de Cycle de Vie quantitative complète n'a jamais été achevée, il est présomptueux d'affirmer que ce type d'analyse procure des données quantitatives rigoureuses [4]. Une chose demeure cependant certaine, c'est que ce type d'outil a su développer un cadre de fonctionnement duquel d'autres méthodes ont pu, et pourraient encore émerger [17].

Un sondage réalisé par Keith Weitz [13] auprès de l'industrie, des écoles, des gouvernements et des firmes de consultation, fait ressortir la présence d'un consensus autour du fait que les ACVS devraient comprendre les quatre éléments de base d'une ACV : but et limite de l'étude, analyse des inventaires, analyse des impacts et interprétation de l'analyse.

La recherche de Keith Weitz a aussi fait ressortir la nécessité de restreindre les frontières du système à analyser afin de réduire la quantité de données à recueillir. Finalement, cette recherche a fait valoir la nécessité de considérer autant les données de type qualitatives que quantitatives.

Janin [9] a identifié cinq avenues pour la simplification de l'ACV:

1. En se limitant à l'étape d'inventaire de la méthodologie (écobilan);

2. En se limitant à un seul critère (approche mono critère) : la consommation de ressources - matière ou énergie l'effet de serre, l'eutrophisation de l'eau ou autre; 
3. En se focalisant sur certains problèmes environnementaux, et ce, sur des étapes particulières du cycle de vie ( l'Évaluation semi-qualitative du Cycle de Vie [ESQCV]: Grille d'évaluation simplifiée et qualitative du Cycle de Vie);

4. En ne tenant compte, lors d'analyses comparatives, que de la phase où les produits sont susceptibles d'avoir des impacts différents;

5. En simplifiant la tâche de collecte de données en ne tenant compte que des données bibliographiques ou génériques.

Ces alternatives de simplification sont spécifiques aux outils d'évaluation d'impacts environnementaux à prédominance quantitative bien qu'elles restent applicables aux outils d'évaluation à prédominance qualitative.

Afin de pouvoir mener à terme notre analyse, nous avons ciblé deux méthodes reconnues par la communauté scientifique et ayant su prouver leur capacité à évaluer l'impact environnemental des produits tout au long de leur cycle de vie. Soit une méthode de type qualitative (ERPA) et une méthode de type quantitative (Écoindicateur99).

\section{$\underline{\text { La méthode ERPA }}$}

En 1993, Thomas E. Graedel et Allenby ont été approchés par AT\&T pour développer une méthode d'évaluation d'impact environnemental. Depuis, cette méthode a été appliquée dans les études de cas suivantes: Graedel (1997), Graedel et Nakaniwa (2002) ainsi que Graedel et Saxton (2002). Cette méthode est également connue sous l'acronyme ERPA Environmentally Responsible Product Assesment.

Identifiée comme étant une approche d'évaluation environnementale de type matriciel et de nature qualitative, sa caractéristique principale est qu'elle s'appuie sur une matrice de deux axes de 5 x 5 . Un axe prend en considération les phases du cycle de vie tandis que l'autre se rapporte aux préoccupations environnementales. Cette méthode permet d'évaluer des produits, des procédés, des bâtiments, des services et des infrastructures.

La méthode consiste à noter l'impact environnemental de chacune des cinq phases du cycle de vie (extraction des ressources, fabrication du produit, emballage, transport, utilisation et fin de vie) en relation avec la sélection de cinq critères environnementaux (choix de matériaux, consommation d'énergie, déchets solides, déchets liquides et déchets gazeux). Le tableau 1 présente un exemple du questionnaire utilisé au moment de la collecte de données. Chacun des éléments de la matrice se voit attribuer un score de 4 (situation défavorable à l'environnement) à 0 (situation favorable à l'environnement). La note maximale que peut obtenir un élément de la matrice est de 4 pour un total de 20 par axe sur une note globale de 100 .
TABLEAU 1.

Exemple de questionnaire pour choix des matériaux/pré-production

Choix des matériaux 1,1_Énoncés applicables

Si une de ces conditions s'applique : 4

La composition chimique des matériaux, composantes, sous-systèmes achetés de fournisseurs n'est pas connue (ou connue partiellement).

L'achat aux fournisseurs de matériaux contenant des substances rares, toxiques ou radioactives alors qu'il existe des alternatives (ces éléments sont les suivants : $\mathrm{Sb}, \mathrm{Be}, \mathrm{B}, \mathrm{Co}, \mathrm{Au}, \mathrm{Cr}, \mathrm{Hg}, \mathrm{Pt}, \mathrm{Ir}, \mathrm{Os}, \mathrm{Rh}$, Ag, Th, U...).

Si une de ces conditions s'applique : 0

Aucun matériau vierge n'est utilisé dans les composantes ou soussystèmes achetés aux fournisseurs.

Si les énoncés précédents ne s'appliquent pas, octroyer 1, 2 ou 3 selon le degré de conformité aux énoncés suivants :

Le produit est-il conçu de façon à minimiser l'utilisation de matériaux, provenant de fournisseurs, contenant des substances rares, toxiques ou radioactives?

Le produit est-il conçu de façon à maximiser l'utilisation de matériaux recyclés?

\begin{tabular}{l|ccccc}
\hline TABLEAU 7.2 The Environmentally Responsible Product Assessment Matrix \\
\cline { 2 - 6 } & \multicolumn{5}{c}{ Environmental Stressor } \\
\cline { 2 - 7 } Product Life-Cycle Stage & $\begin{array}{c}\text { Materials } \\
\text { Choice }\end{array}$ & $\begin{array}{c}\text { Energy } \\
\text { Use }\end{array}$ & $\begin{array}{c}\text { Solid } \\
\text { Residues }\end{array}$ & $\begin{array}{c}\text { Liquid } \\
\text { Residues }\end{array}$ & $\begin{array}{c}\text { Gaseous } \\
\text { Residues }\end{array}$ \\
\hline Premanufacture & 1,1 & 2,1 & 3,1 & 4,1 & 5,1 \\
Product Manufacture & 2,1 & 2,2 & 3,2 & 4,2 & 5,2 \\
Product Delivery & 3,1 & 2,3 & 3,3 & 4,3 & 5,3 \\
Product Use & 4,1 & 2,4 & 3,4 & 4,4 & 5,4 \\
Refurbishment, Recycling, Disposal & 5,1 & 2,5 & 3,5 & 4,5 & 5,5 \\
\hline
\end{tabular}

Fig. 1. exemplaires de la matrice d'entré des données [3]

Les résultats de l'analyse obtenus à l'aide de la matrice ERPA sont ensuite insérés dans une cible Fig.2. afin de faciliter l'interprétation des résultats.

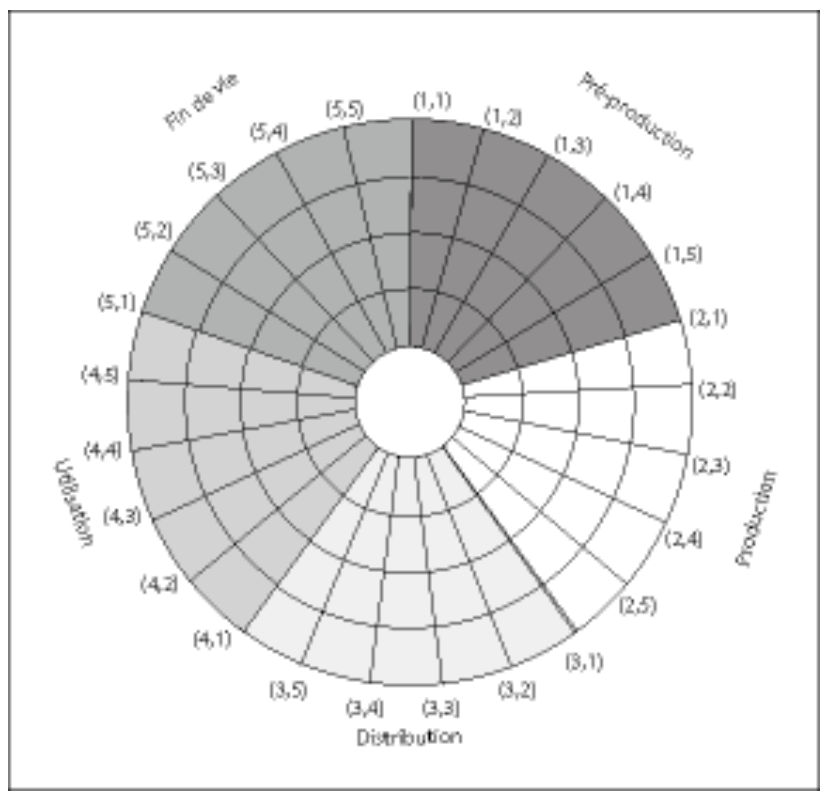

Fig.2. Cible des résultats proposée par Graedel pour l'interprétation de la méthode ERPA. [3] 


\section{La méthode Éco-indicateur99}

Cette méthode a été mise au point par le groupe Pré Consultant qui regroupe des chercheurs néerlandais et suisses. Cette méthode a été conçue suite à une demande du Ministère de l'Environnement néerlandais.

L'outil Éco-indicateur99 est une méthode d'évaluation d'impact environnemental, de nature quantitative, orientée sur les dommages causés par l'émission de substances néfastes à l'environnement lors du processus industriel. Cette méthode cherche à unifier les résultats de l'analyse en un indicateur unique.

L'objectif principal de cet outil était de développer une méthode d'analyse environnementale pour les produits. Il en résultait une liste d'une centaine d'indicateurs propres aux matériaux et procédés les plus communs. L'utilisation de ces indicateurs permet au designer d'employer une analyse de cycle de vie des impacts environnementaux du produit qu'il crée.

Afin d'aider les designers et les gestionnaires à utiliser l'Éco-indicator99, le groupe Pré-Consultant propose un guide d'utilisation d'indicateurs environnementaux standardisés qui s'appuient sur les valeurs de l'Écoindicateur99. Le programme Éco-it, aussi développé par Pré-consultant, permet de faciliter la tâche de l'utilisateur durant l'évaluation d'un produit.

Le manuel d'opération proposé aux designers leur permet de prendre en considération les cinq étapes d'exécution à suivre afin de conduire une analyse fiable à l'aide des Éco-indicateur99 :

1. Objectif et définition du champ d'études

2. Définition du cycle de vie

3. Inventaire des matériaux et procédés

4. Prise en note des données

5. Interprétation des résultats

La phase de l'inventaire consiste à :

- Déterminer l'unité fonctionnelle.

- Quantifier tous les éléments présents dans l'arbre d'inventaire.

- Émettre des suppositions pour les données manquantes

Dans le cadre général d'une ACV, la description du produit se fait en termes d'unité fonctionnelle. Une quantité peut alors être inscrite dans chacun des éléments de l'arbre de cycle de vie basé sur cette unité fonctionnelle et sur les données recueillies lors d'une recherche de données empiriques préalables.

La phase de prise en compte de donnés sert à :

- Prendre en note les informations relatives aux matériaux et procédés, pour ensuite les inscrire sur la fiche élaborée à cette fin.
- Trouver les indicateurs propres aux éléments indiqués sur la fiche.

- Calculer les impacts en multipliant les quantités trouvées par la valeur de l'indicateur.

Afin de faciliter la tâche aux designers, une fiche de calcul a été élaborée. En ce sens, le groupe Pré-Consultant a mis au point le programme Éco-it qui, tout comme la fiche, permet de cumuler et de calculer les données recueillies. La Fig.3. présente le modèle de la grille d'inventaire proposée pour entreprendre l'évaluation environnementale d'un produit.

Une fois la phase d'inventaire achevée et l'évaluation complétée, il ne reste à l'utilisateur qu'à représenter de façon compréhensible les résultats obtenus. La Fig.4. est la méthode que nous avons privilégiée.

Fig.3. Grille d'inventaire proposée par le groupe Pré-Consultant [23]

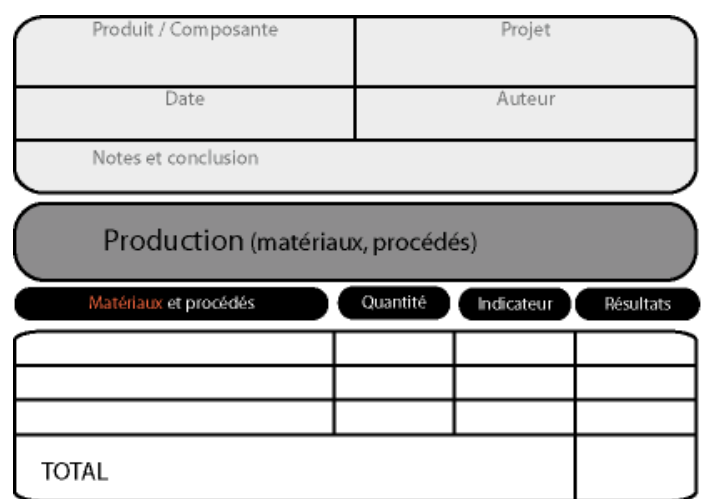

Usage (transport, énergie et matériaux auxilières)

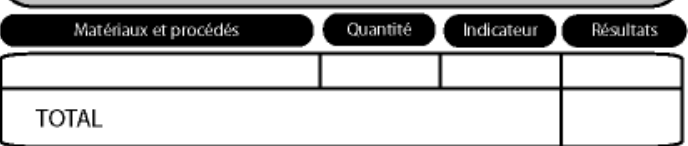

Fin de vie (pour chaque type de matériaux)

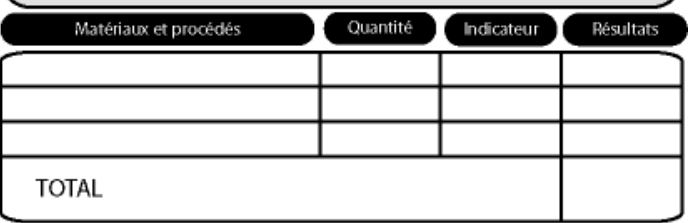

TOTAL 
Fig.4. Modèle de représentation des résultats

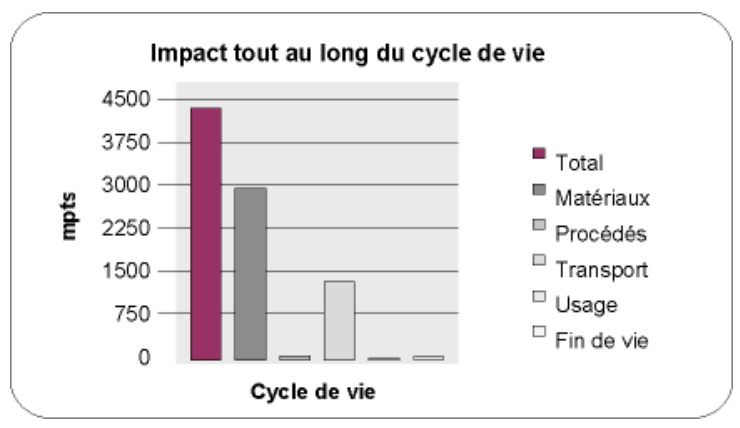

\section{Résultats des analyses}

Dans cette partie de notre travail, nous exposerons les résultats de nos deux analyses d'impact. Nous présenterons ces derniers de façon à démontrer les résultats obtenus pour chacune des étapes du cycle de vie. Le système produit (arbre de cycle de vie) a été réalisé lors de la phase d'inventaire des deux méthodes. Par contre, la majorité des données identifiées ont été recueillies lors de la phase d'inventaire de l'Ecoindicateur 99.

\section{$\underline{\text { Les résultats obtenus avec ERPA }}$}

Les résultats obtenus à l'aide de la méthode ERPA sont illustrés dans la Figure 5 et dans le Tableau 2.

Lors de la réalisation de notre étude, et particulièrement au moment de l'analyse de l'étape de préproduction, il nous a été possible de constater des insuffisances au niveau du temps accordé à l'analyse, ainsi qu'au niveau de la quantité d'informations retenues. Ceci s'explique notamment par le fait que la collecte d'informations sur l'ensemble des spécificités des composantes utilisées pour la fabrication du meuble s'est avérée plus ardue que prévu. L'absence de collaboration des fournisseurs explique par ailleurs le fait que de nombreuses données sont issues de la littérature existante ainsi que des sites Web d'autres fournisseurs offrant des produits similaires.
Figure5

Les résultats obtenus à l'aide de la méthode ERPA

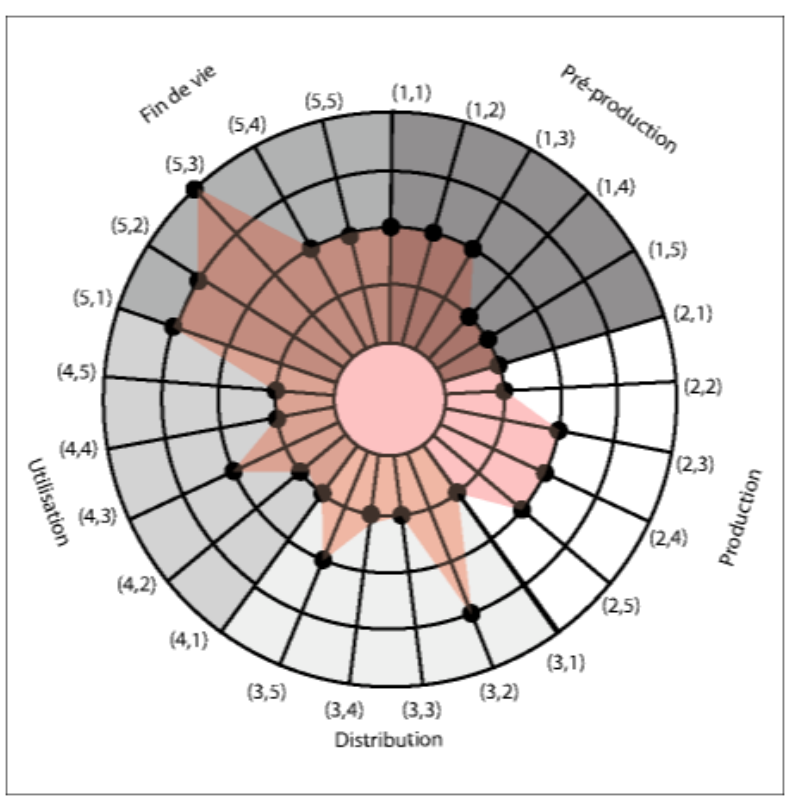

Les conclusions de notre analyse d'impact avec cette méthode sont les suivantes :

- Le produit a un impact considérable sur la quantité de déchets solides qu'il génère en fin de vie.

- La quantité de déchets solides générée par ce produit tout au long de cycle de vie est significative.

- L'impact le plus important lors de la production est la quantité de déchets générés.

- La distribution du produit nécessite une importante demande énergétique.

- L'utilisation de ce produit n'a pratiquement pas d'impact.

TABLEAU 2.

Résultats obtenus avec la matrice d'impact proposée par Geaedel

Cycle de vie

Cycle de vie du produit

Cholx

Energie

de matériaux, utilisé , Solides, Liquides Gazeux

Risidus

Residus

Residus

\begin{tabular}{|l|c|c|c|c|c|c|}
\hline Pré-production & 2 & 2 & 2 & 1 & 1 & $8 / 20$ \\
\hline Production & 1 & 1 & 2 & 2 & 2 & $8 / 20$ \\
\hline Distribution & 1 & 3 & 1 & 1 & 2 & $8 / 20$ \\
\hline Utilisation & 1 & 1 & 2 & 1 & 1 & $8 / 20$ \\
\hline Fin de Vie & 3 & 3 & 4 & 2 & 2 & $14 / 20$ \\
\hline TOTAL & $8 / 20$ & $10 / 20$ & $11 / 20$ & $7 / 20$ & $8 / 20$ & $46 / 100$ \\
\hline
\end{tabular}


$\underline{\text { Les résultats obtenus avec Éco-indicateur99 }}$

Les résultats obtenus à l'aide de la méthode Ecoindicateur99 sont illustrés dans la figure 6 ainsi que dans le figure7.

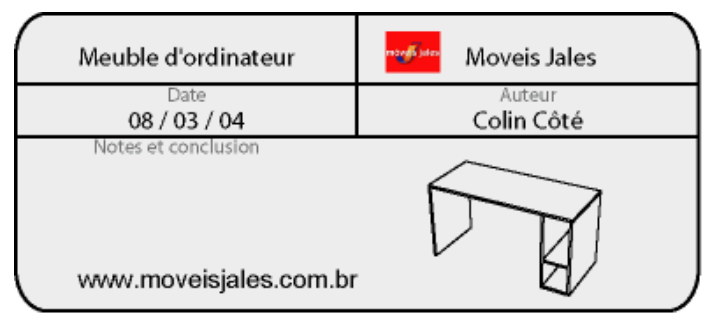

Production (matériaux, procédés)

\begin{tabular}{|c|c|c|c|}
\hline Matériat & & ind & Résultats \\
\hline $\operatorname{MDF}(2,5 \mathrm{~cm})$ & $57,9960 \mathrm{~kg}$ & 39 & 2261.844 \\
\hline $\operatorname{MDF}(1,5 \mathrm{~cm})$ & $13,4270 \mathrm{~kg}$ & 39 & 523.653 \\
\hline $\operatorname{MDF}(0,3 \mathrm{~cm})$ & $0,9370 \mathrm{~kg}$ & 39 & 36.543 \\
\hline Acier & $1,4238 \mathrm{~kg}$ & 86 & 122.447 \\
\hline HDPE & $0,0310 \mathrm{~kg}$ & 330 & 10.230 \\
\hline PET & $0,0590 \mathrm{~kg}$ & 380 & 22.420 \\
\hline Peinture ${ }_{\boldsymbol{0}_{(0)}}$ & 0,761 & - & - \\
\hline Colle à bois & $2 \mathrm{~kg}$ & - & - \\
\hline Gougeons & $0,0290 \mathrm{~kg}$ & 6.6 & 0,1914 \\
\hline Collage bois $x_{(1)}$ & $3,135 \mathrm{~m}^{2}$ & - & - \\
\hline Coupe du bois $x_{x p 2}$ & $4,252 \mathrm{dm}$ & 6.4 & 27.213 \\
\hline Perçage bois $x_{2} 2$ & $0,712 \mathrm{dm}^{3}$ & 6.4 & 4.557 \\
\hline HDPE moulage par injection & $0,0310 \mathrm{~kg}$ & 21 & 0.651 \\
\hline Pliage de l'acier & $3,2 \mathrm{~m}$ & 0,00008 & 0.00026 \\
\hline Perçage acier & $0,0045 \mathrm{~m}^{2}$ & 0,00006 & 0.00001 \\
\hline Acier mouler sous pression & $0,065 \mathrm{~kg}$ & - & - \\
\hline Peinture & $4,76 \mathrm{~m}^{2}$ & - & . \\
\hline PET termoformage & $0,0590 \mathrm{~kg}$ & 9.1 & 0.5369 \\
\hline TAL & & & 3008, \\
\hline
\end{tabular}

Usage (transport, énergie et matériaux auxilières)

\begin{tabular}{|l|c|c|c|}
\hline \multicolumn{1}{|c|}{ Matériaux et procédés } & Quantité & Indicateur & Résultats \\
\hline Camion de livraison $<3.5 \mathrm{t}$ & $30 \mathrm{~km}$ & 140 & 1200 \\
\hline TOTAL & & $\mathbf{1 2 0 0}$ \\
\hline
\end{tabular}

Fin de vie (pour chaque type de matériaux)

\begin{tabular}{|c|c|c|c|}
\hline Materiaux et procé & Quantité & ndicat & Résultats \\
\hline Acier & $1,4238 \mathrm{Kg}$ & 1,4 & 1.993 \\
\hline $\operatorname{MDF}(2,5 \mathrm{~cm})$ & $57,9960 \mathrm{Kg}$ & & - \\
\hline $\operatorname{MDF}(1,5 \mathrm{~cm})$ & $13,4270 \mathrm{Kg}$ & - & - \\
\hline $\operatorname{MDF}(0,3 \mathrm{~cm})$ & $0,9370 \mathrm{Kg}$ & - & - \\
\hline HIPS (Roues) & $0,0590 \mathrm{Kg}$ & 4,1 & 0.242 \\
\hline PVC (Dissp. mont.) & $0,0310 \mathrm{Kg}$ & 2,8 & 0.088 \\
\hline \multicolumn{3}{|l|}{ TOTAL } & 2.323 \\
\hline Volume & $0,1076 \mathrm{m3}$ & 140 & 15,064 \\
\hline \multicolumn{3}{|l|}{ TOTAL } & 15,064 \\
\hline \multicolumn{3}{|l|}{ TOTAL } & 4223.074 \\
\hline
\end{tabular}

Fig.6. Résultat analyse avec Eco-indicator'99
L'analyse d'impact avec cette méthode s'est relativement bien déroulée. Il est toutefois important de souligner que la restriction liée à la quantité d'Ecoindicateurs disponibles ne nous laissait d'autres choix que d'élaborer certaines suppositions. L'absence d'indicateurs, pour le processus de fabrication de la coupe $\mathrm{du}$ bois, nous a obligé à suivre les recommandations du IHBO, et à assumer le fait que l'impact de ce processus demeure semblable à celui du perçage du plastique [18].

Contrairement à la méthode ERPA, aucun mode de représentation des résultats n'est proposé par l'Écoindicateur99. Pour remédier à ce problème, nous avons utilisé un mode de représentation des résultats sous forme de tableau Fig.7.

Les conclusions de notre analyse d'impact avec cette méthode sont les suivantes :

- Le produit a un impact considérable lors de la phase de préroduction, principalement lors du choix des matériaux (MDF).

- Lors de la phase de fabrication du produit, l'impact le plus important se situe au niveau de la coupe du bois.

- La distribution du produit nécessite une importante demande énergétique.

- $\quad$ En fin de vie, le produit a un impact plus grand si on privilégie le volume au détriment du poids.

- L'utilisation de ce produit n'a pratiquement pas d'impact.

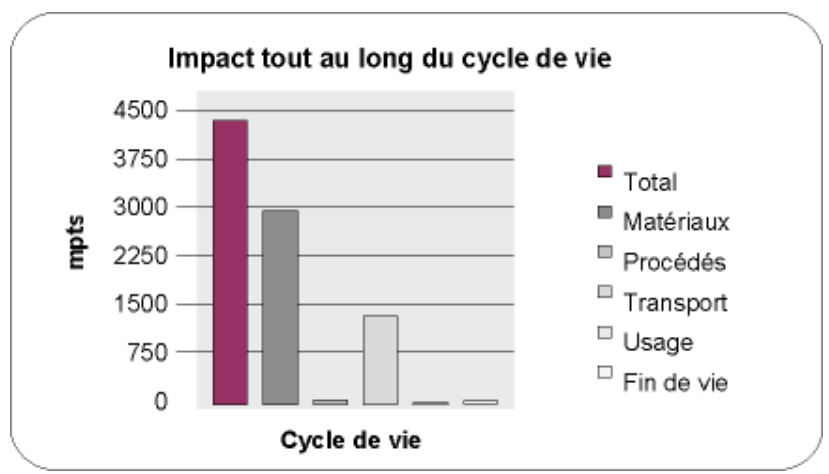

Fig.7. Résultat analyse avec Eco-indicator'99

Les résultats et les conclusions tirés des deux méthodes sont différents. Les deux méthodes ont prouvé que l'étape du cycle de vie ayant le moins d'impact est celle de l'utilisation. Les conclusions issues de la méthode ERPA ont démontré que l'objet avait un impact majeur en fin de vie. L'Éco-indicateur99 a, quant à lui, démontré que l'impact majeur est associé à la sélection des matériaux (étape de préroduction). Le Tableau 3. illustre l'ensemble des impacts identifiés par les deux méthodes d'analyse. Les résultats ont été convertis en pourcentage afin de permettre de mieux visualiser les différences. 
TABLEAU 3.

Comparaison du cycle de vie des deux méthodes d'analyse d'impact.

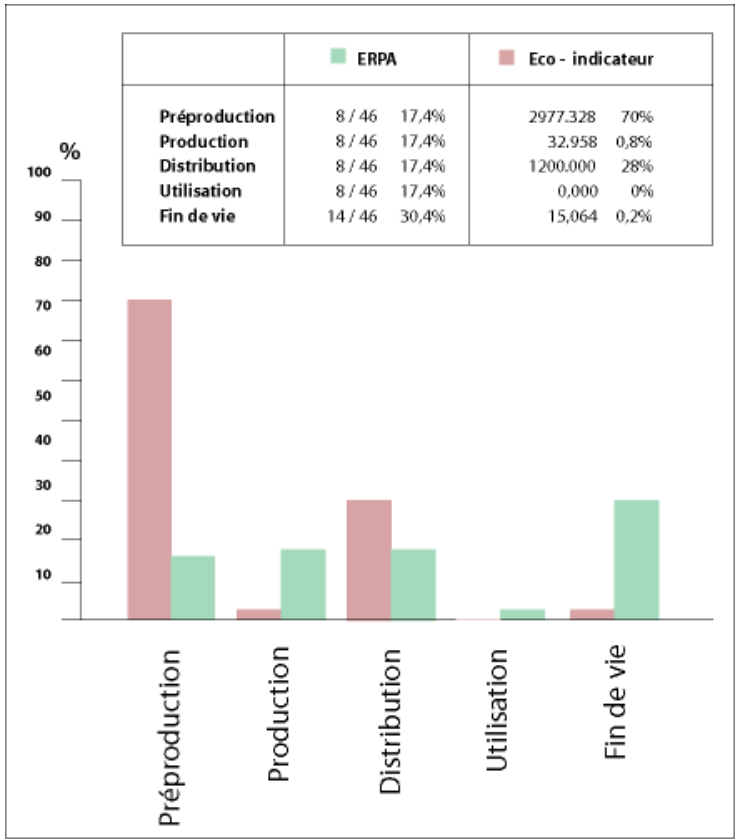

\section{4. Évaluation des méthodes}

Les résultats de l'évaluation comparative sont présentés dans le Tableau 4. Les résultats obtenus lors de l'analyse d'impact avec ces deux méthodes nous fournissent différents types d'informations. Chaque élément de la grille d'évaluation fait référence à une question. Si une question obtient une appréciation positive une note de 5 lui est attribuée si l'appréciation est négative une note de 1 est accordé pour une note totale de 100 .

Nous présenterons les résultats de notre évaluation de la façon suivante: pour chaque méthode, nous identifierons les points faibles et les points forts tout au long des trois étapes de l'analyse d'impact soit: phase d'inventaire, évaluation des impacts et interprétation des résultats.

\subsection{Phase d'inventaire :}

L'utilisation des deux méthodes a fait ressortir le fait que la méthode Éco-indicateur99 ne permet pas d'être modifiée ou ajustée selon les besoins de l'évaluateur. En effet, ce dernier doit se fier aux indicateurs proposés et travailler avec eux. Cette rigidité a le démérite de minimiser le temps nécessaire à la cueillette des informations relatives à l'inventaire. Néanmoins, cette rigueur facilite la compréhension du processus et ne laisse pas l'évaluateur dans l'interrogation, lui permettant ainsi d'obtenir aisément l'information recherchée.

La méthode ERPA a pour avantage de laisser plus de liberté à l'évaluateur quant aux choix des facteurs d'impact qu'il désire évaluer et d'élaborer lui-même son questionnaire de cueillette de données. La méthode permet donc une très grande flexibilité quant au genre d'analyse que l'on veut mener. Par contre, il a clairement été remarqué que cette méthode laisse parfois l'évaluateur perplexe face au choix des informations qu'il recherche. Il a été noté que la recherche d'informations pour la phase de pré production a nécessité plus de la moitié du temps nécessaire à l'analyse. $\mathrm{La}$ difficulté à obtenir les renseignements auprès des fournisseurs a pu être contournée par l'entremise d'autres fournisseurs commercialisant des produits similaires.

Il a aussi été noté que certaines données utilisées avec la méthode Eco-indicateur99 auraient pu être utilisées pour répondre à certaines questions du questionnaire d'ERPA.

TABLEAU 4.

Résultats de l'évaluation de méthode d'analyse.

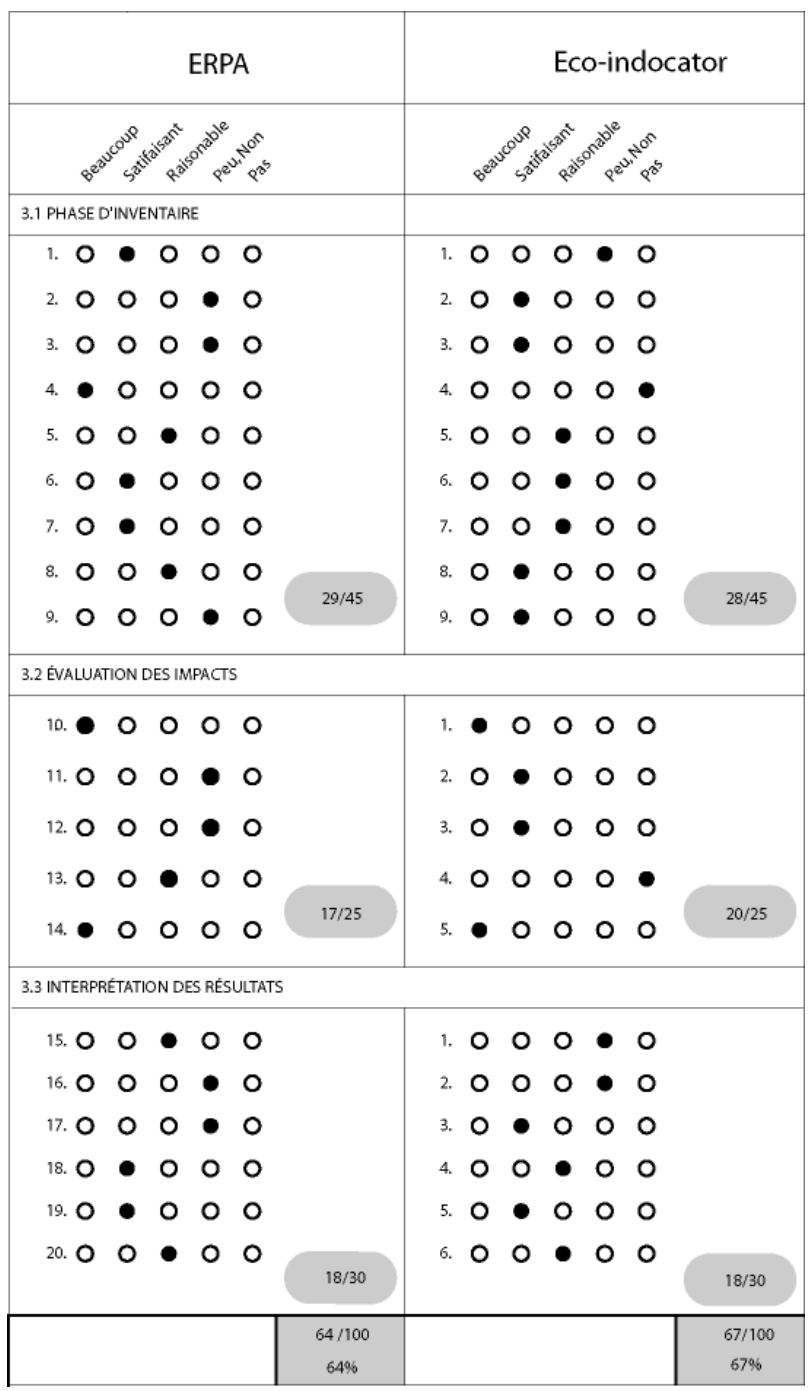

4.2 Évaluation des impacts.

Une des particularités de la méthode ERPA est qu'elle permet à l'évaluateur de pourvoir obtenir deux types de résultats finaux. Premièrement, cette méthode permet à l'évaluateur de dresser un portrait des impacts sur 
l'ensemble du cycle de vie. Deuxièmement, elle permet à l'évaluateur de pouvoir identifier quelle catégorie d'impact a le plus d'incidence tout au long du cycle de vie de l'objet. La principale difficulté qui a été identifiée est que l'évaluateur peut rencontrer lors de l'attribution d'un résultat pour un des éléments de la matrice ex : préproduction - déchets solides. Cette méthode permet d'obtenir un résultat unique pour l'ensemble de l'évaluation.

Une fois l'inventaire fait, la méthode Éco-indicateur99 se fait presque par elle-même. Il suffit à l'évaluateur de multiplier l'indicateur par l'information $(\mathrm{kg}, \mathrm{lb}, \mathrm{dm}, \ldots)$ recueilli lors de l'inventaire. Cette étape de l'analyse se fait très rapidement. Les problèmes qui ont été rencontrés sont la limitation quant à la quantité d'indicateurs disponibles. Cette situation a pour effet de confronter l'évaluateur à laisser de côté l'information recueillie ou le forcer à trouver une alternative à l'indicateur manquant. Comme pour la méthode ERPA cette méthode permet à l'évaluateur d'arriver à octroyer un résultat unique pour l'ensemble de son évaluation.

\subsection{Interprétation des résultats}

Lorsque Greadel élabora la méthode ERPA, une attention particulière fut portée sur la façon dont les résultats finaux seraient présentés. La méthode ERPA dispose d'un ingénieux système qui permet de convertir les données qualitatives en résultat quantitatif. Greadel a proposé l'utilisation d'une cible d'impact. Cet outil s'est avéré être très efficace pour la visualisation rapide des impacts, sur l'ensemble du cycle de vie de l'objet. Le temps nécessaire pour cette phase de l'analyse est relativement rapide.

La méthode Éco-indicateur99 ne dispose pas de système prédéfini de visualisation. Le principal outil de visualisation est la différence numérique des résultats obtenus pour chaque phase du cycle de vie. Il est donc laissé à la discrétion de l'évaluateur de présenter ses résultats comme il lui convient. Pour notre part, nous avons privilégié un système cartésien, sur deux axes, à l'intérieur duquel l'axe des $\mathrm{X}$ représente les cinq phases du cycle de vie et l'axe des Y les résultats en millipoints, unité utilisée pour l'analyse. Si l'évaluateur désire présenter ses résultats sur un graphique, il lui faudra prévoir du temps pour la construction de son graphique.

Les deux méthodes utilisées ont su cibler différents problèmes environnementaux tout au long du cycle de vie de l'objet. Par contre, la méthode ERPA a nécessité un peu plus de temps que la méthode Éco-indicateur99.

\section{Avantages et Inconvénients}

Méthode Éco-indicateur99

\begin{tabular}{|c|c|}
\hline Avantages & Inconvénients \\
\hline - Elle permet d'attribuer une & - La liste des éco- \\
\hline
\end{tabular}

valeur numérique des impacts environnementaux du produit et des processus sans faire l'usage de logiciel.

- Disponible sur le Web et entièrement gratuite.

- Elle permet de dresser un portrait complet du cycle de vie du produit.

- Simple et efficace

Elle permet une comparaison entre deux propositions ou deux systèmes produits différents.

\section{Méthode ERPA}

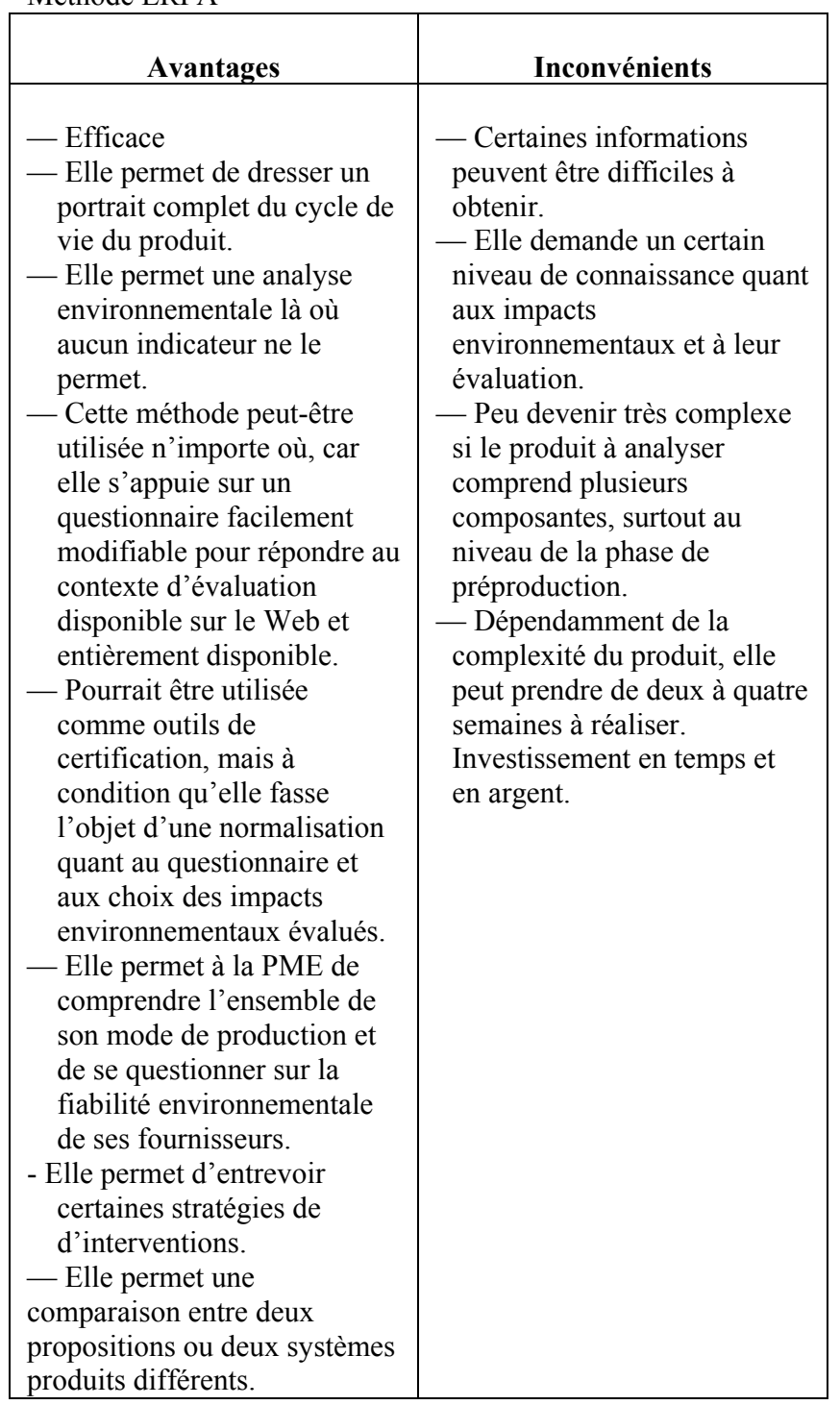

\section{$\underline{\text { La méthode Eco-indicateur'99 }}$}

La fiabilité de cette méthode est assurée par la reconnaissance scientifique dont elle jouit. Par contre, les résultats qu'elle produit sont basés sur des indicateurs qui ont été produits pour répondre aux conditions européennes. Néanmoins, elle permet au concepteur de dresser un modèle fiable de l'ensemble du cycle de vie du produit qu'il évalue et de pouvoir cibler certains éléments d'intervention possible. Il serait intéressant de voir quels 
seraient les résultats d'une analyse s'appuyant sur des indicateurs propres à la situation brésilienne, pour ensuite les comparer avec les résultats obtenus avec des indicateurs propres à la situation européenne.

Cette méthode demeure très accessible, ne demandant pas un très haut niveau de connaissance quant aux impacts environnementaux, mais exigeant en revanche un niveau de connaissance élevé quant à l'évaluation environnementale. Son utilisateur devra cependant bien comprendre l'ensemble du système produit que la fabrication que son produit génère. Cette méthode est disponible sur le site Web du groupe néerlandais PréConsultant [22]. Un guide d'utilisation très bien construit est également disponible.

Cette méthode répond bien au contexte des PME, car elle demande peu de temps d'utilisation tout en ne requérant qu'une connaissance superficielle de toute la méthodologie sous-tendant la méthode. Il est donc raisonnable d'affirmer que ce type d'outil peut être employé dans le contexte de la conception par les PME. Ainsi, avoir recours à ce type d'outil s'avère entièrement faisable pour une PME.

\section{$\underline{\text { La méthode ERPA }}$}

Cette méthode a fait l'objet de plusieurs articles et a été utilisée dans plusieurs études de cas. Elle a aussi fait l'objet de plusieurs publications et est reconnue par la SETAC [7] comme une bonne alternative à l'analyse de cycle de vie complète (ACV). Cette méthode se base sur un questionnaire qui a été construit en fonction des stratégies de conception en design pour l'environnement (DFE), proposé par Geaedel. Elle permet à l'évaluateur d'analyser l'ensemble du cycle de vie d'un produit selon des critères environnementaux prédéfinis par ce dernier. Cette flexibilité rend cette méthode très versatile, ce qui a pour résultat de rendre l'évaluation plus appropriée à certaines conditions. Le désavantage lié à cette souplesse est que dans l'éventualité d'une mauvaise utilisation ou interprétation, les résultats obtenus peuvent s'avérer peu fiables.

Cette méthode s'adresse à des gens qui ont une certaine connaissance de l'évaluation environnementale. Par contre, il est possible de pouvoir apprendre à utiliser cette méthode en se procurant le livre de Graedel intitulé Streamlined Life-Cycle Assessment [3]. Le contenu du livre est bien expliqué et propose une multitude d'exemples, dont plusieurs s'attardant à des modèles de questionnaire pour la réalisation de la phase d'inventaire. Cette méthode est cependant moins accessible que la méthode Éco-indicateur.

La méthodologie proposée convient bien au contexte dans lequel évoluent les PME brésiliennes. Elle permet d'être appliquée par les fabricants de produits ainsi que par les personnes désirant évaluer l'impact du service qu'ils rendent, ou celui d'une installation (construction) dont ils disposent.

\section{Discussion et conclusion}

La situation actuelle des PME brésiliennes nous laisse croire que l'introduction de ce type d'outil au sein de ces entreprises n'est pas pour bientôt [14] [1]. Nous croyons cependant que ce type d'outil pourrait devenir un atout majeur pour les PME qui désirent concurrencer sur le marché local, national ou international.

L'intégration de ce type d'outil d'analyse devra se faire graduellement par la sensibilisation d'abord faite auprès des propriétaires d'entreprises, de leurs employés ainsi qu'auprès des fournisseurs.

Nous croyons qu'un programme de consultation et d'éducation pourrait permettre de former certains membres d'entreprise à comprendre et utiliser ce type d'outil. La mise en place d'un réseau sensibilisé aurait l'avantage d'assurer l'existence d'une production environnementale des plus intéressante qui pourrait à son tour engendrer des retombés économiques considérables, tout en permettant aux fabricants une reconnaissance nationale et internationale.

Nos conclusions nous portent à croire que dans un premier temps, la méthode Éco-indicateur99 devrait être privilégiée dans sa forme actuelle, malgré l'absence d'indicateurs propres à la situation brésilienne. Toutefois, une attention particulière pourrait être apportée à un mode de représentation des résultats plus explicite. Sur une période à plus long terme, la méthode ERPA devrait être privilégiée. Cette méthode a l'avantage de s'appuyer sur un questionnaire permettant de mieux entrevoir les stratégies environnementales potentiellement envisageables dans l'optique d'une amélioration du système produit et de son design. De plus, elle s'adapte plus facilement aux différentes situations que pourrait rencontrer l'évaluateur.

\section{Remerciements}

Les auteurs voudraient également remercier l'aide financière du FAQDD, de l'OQAJ, du gouvernement du Québec et de son programme de Bourse de Mobilité, de CAPES et d'IFM-CNPq du gouvernement du Brésil. Les auteurs tiennent aussi, remerciez: Sylvain Plouffe et Maxime Thibault du Laboratoire-écodesign. Un grand merci au plus Grand Fou des Grands fous Alexis De Lancer ainsi qu'à Colette Robitaille et Daniel Côté. 


\section{Références}

[1] G J Costa and R P Gouvinhas., "Ecodesign strategies and the product developpement process within Northeast SME Brazilian companies - International Conference on Engineering Design ICED 03 Stockholm, August 19-21, 2003

[2] Costa G.J., and Gouvinhas RP., "The Utilisation of Ecodesign Practices within Brazilian SME Companies". Proceedings of Toward Sustainable Product Design -7 th International Conference, London, UK, July -2002.

[3] Graedel TE (1998): Streamlined Life-Cycle Assessment. New Jersey. Prentice Hall Inc.

[4] Graedel TE, Allenby BR (1995): Industrial Ecology. New Jersey. Prentice HallBRODHAG, Christian (2001), Glossaire pour le développement durable, France, Agora 21, 62pages

[5] UNEP (United Nations Environment Programme) / TU Delf / Rathenau Institute (1997), Eco - Design: Promising Approach to Sustainable Production and Consumption, (Stevenage, UK: EarthPrint, www.earthprint.com), 346pages

[6] SETAC (Society of Environmental Toxicology and Chemistry), (2001) Streamlined Life Cycle Assessment Study, Environmental Resources Management, Oxford. 18 pages

[7] SETAC (Society of Environmental Toxicology and Chemistry), (2001) Streamlined Life Cycle Assessment: A Final Report from the SETAC North America Streamlined LCA Workgroup, SETAC \& SETAC Foundation for Environmental Education. 31pages

[8] Hunt RG. Boguski Tk, Weitz K., Sharma A (1998): Case studies Examining LCA streamlining Techniques Int J.LCA 3(1) $36-42$

[9] JANIN, Marc, Démarche d'éco-conception en entreprise Un enjeux : Construire la coherence entre outils et processus, 2001, Thèse de doctorat ENSAM,

[10] Laurence Toffoletto, Julie-Anne Chayer, Pascal Lesage (2003), PROTOCOLE STANDARD OPÉRATIONNEL Guide CIRAIG (Élaboration générale d'une Analyse du Cycle de Vie (ACV) simplifiée), Montréal, CIRAIG, PSO_A 20

[11] LEWIS, Helen \& GERTSAKIS, John, 2001, Design + Environment, UK, Greenleaf Publishing Limited. 198 pages

[12] Todd, J.A., Streamlining, in environmental life cycle assessment, M.A Curran, ed. (New York: Mc Graw-hill, 1996)

[13] Weitz K.A ., C. Hildebrand, R.Hunt, A. Sharma and L. Hogan; Accurate Streamlined Life Cycle Assessment Is it Possible? (1996)

[14] SEBRAE. Available in: www.sebrae.com.br. Accessed in: January, 27th 2003.
[15] CIRAIG: Centre Interuniversitaire de Référence sur l'Analyse, l'Interprétation et la Gestion du cycle de vie des produits, procédés et services. www.polymtl.ca/ciraig/

[16] Hochschorner1, E and Finnveden G Evaluation of Two Simplified Life Cycle Assessment Methods, Int J LCA 8 (3) 2003 page 120

[17] AFNOR Grisel, L. \& Duranthon, G., Guide D'écoconception, France, 2001, 128pages

[18] IHBO, A Practical Manual Of Ecodesign, Basque Governement of Territorial Organisation, housing and the Environnement. May 2001, 137pages.

[19] Geodkoop M.J.; Spriensma R.S.; The Eco-indicator99, Methodology report, A damage oriented LCIA Method; VROM Report -------, Den Haag, 1999 [132 pages]

[23] Geodkoop M.J.; Spriensma R.S.; The Eco-indicator99, Manual for designers, Avril 2000, 22pages

[20] Meuble Jales: www.moveisjales.com.br

[21] Potidesign: http://www.potidesignufrn.com

[22] Pré-Consultant: http://www.pre.nl

\section{Rappel}

Nous donnons la permission au CDEN de publier ce manuscrit.

\section{Correspondances}

Colin Côté

Faculté de l'aménagement de l'Université de Montréal

École de design industriel

2940, Chemin Côte Ste-Catherine

Montréal (Québec),Canada H3C 1B9

Téléphone : 514 343-6111, ext. 5249,

e-mail : Lokinlado@hotmail.com

labotatoire-ecodeisgn@umontreal.qc.ca

Dr. Reidson Pereira Gouvinhas

Universidade Federal do Rio Grande do Norte - UFRN,

PEP/UFRN, Campus Universitário, Caixa Postal 1551, Lagoa

Nova, Natal/RN, 59078-970, Brasil,

Phone: + 5584 2153711; Fax: + 55842153711 ;

e-mail: reidson@ct.ufrn.br 